\title{
The effects of the contrast, spatial frequency, and temporal frequency of a surrounding field upon the detection thresholds of gratings
}

\author{
ALISON BOWLING \\ Monash University, Clayton, Victoria, Australia
}

\begin{abstract}
Three experiments, in which the temporal and spatial characteristics of square-wave gratings surrounding a central test field were varied, are reported. The detection thresholds of 1 -sec presentations of a $5-\mathrm{Hz}$ counterphase flickering .5-cycle/deg (cpd) sinusoidal grating were measured under the different surround conditions. Threshold was found to increase with increasing surround contrast, and to be confined to surround spatial frequencies of $2 \mathrm{cpd}$ and below. Maximum threshold elevation occurred with surround drift frequencies at about $8 \mathrm{~Hz}$, irrespective of spatial frequency. It was concluded that the surround effect is probably due to an inhibitory interaction between transient-type mechanisms in the central visual field and the pheriphery.
\end{abstract}

It has recently been shown that sensitivity to a central low-spatial-frequency test grating is reduced by a moving grating or counterphase flickering blank field surrounding it. The reduction in sensitivity is confined to test spatial frequencies lower than about 2 cycle/deg (cpd) (Bowling, 1985; Green, 1983). The effect occurs both with counterphase flickering test gratings and with briefly pulsed presentations of the test stimuli. Bowling hypothesized that the surround effects would occur only in situations in which "transient" mechanisms were stimulated, since it was thought that temporal modulation of the surround altered the responsiveness of mechanisms with this type of time-course. ${ }^{1}$ Flickering and pulsed presentations of low-spatial-frequency gratings appear strongly to stimulate these mechanisms. The pronounced reduction in sensitivity to low-spatial-frequency flickering or pulsed gratings thus supported the hypothesis. However, Bowling (1985) also observed a low-spatial-frequency sensitivity reduction when the test presentation was a $1-\mathrm{sec}$ cosine temporal envelope, indicating that the same mechanism is perhaps responsive to low spatial frequencies under both flickering and slow onset conditions.

The nature of the surround was also shown to have some influence on sensitivity reduction. A flickering blank field, and a moving low-frequency square-wave grating, both temporally modulated at $6.8 \mathrm{~Hz}$, produced similar effects, but a greater reduction in sensitivity was observed when the orientations of the test and surround gratings were the same than when they differed by $90^{\circ}$. Consequently, it appears that although the sensitivity reduction is partly produced by the temporal modulation of the surround,

This experimental work was made possible by the award of an Australian Research Grants Service grant to the author. The contribution of Daveena Brain in serving as subject is also gratefully acknowledged. The author's mailing address is: $\mathbf{5 3}$ King Street, Bellerive, Tasmania, Australia 7018. there is also an influence of the nature of the surrounding pattern. The experiments to be reported in this paper investigated these effects more fully.

The experiments reported by Bowling (1985) were based on electrophysiological work by Kruger (1980, 1981), who demonstrated that the time-course of cat $Y$ cells becomes more sustained in the presence of peripheral motion. It has been suggested that this effect may be due to nonlinear subunits which apparently influence the activity of $Y$ cells, but not that of X cells (Derrington, Lennie, \& Wright, 1979). It is proposed that the reduction in sensitivity observed in the human psychophysical experiments is the result of the activity of a similar nonlinear network in humans which may also influence the sensitivities of mechanisms with transient time-courses.

This paper reports experiments designed to test this hypothesis. In cats, the nonlinear subunits are responsive to higher spatial frequencies than those that produce the modulated response of Y cells (Derrington et al., 1979; Hochstein \& Shapley, 1976). Their activity saturates rapidly as the luminance of the bright bars (and hence contrast) of an inducing grating increases (Barlow, Derrington, Harris, \& Lennie, 1977; Derrington et al., 1979). If such mechanisms exist in humans, then spatial frequency and luminance (or contrast) should influence these subunits in a manner similar to the way in which they are affected in the cat. Specifically, their activity should saturate with increasing contrast, and they should respond to higher spatial frequencies than those that give rise to a transient type of response.

If the reduction in sensitivity to low-spatial-frequency gratings in the presence of peripheral movement is due to such subunits, then this effect should be influenced by the contrast and spatial frequency of the moving surround. An increase in the contrast of the surround should produce an initial increase in the threshold of a central counterphase flickering low-spatial-frequency grating. This 
should then level off as the response of the hypothetical subunits saturates. There should thus be a nonlinear relationship between surround contrast and test threshold elevation. In addition, the maximum surround spatial frequency that produces the test threshold elevation should be higher than the highest spatial frequency to which the transient mechanism is normally responsive (about $2 \mathrm{cpd}$ ). The following experiments consequently investigate the effects of surround contrast and spatial frequency upon the threshold elevation of a central low-spatial-frequency test grating.

\section{METHODS}

The apparatus and procedure were the same as those described by Bowling (1985). The stimuli were generated by means of a CRT image generator and displayed on an HP131OA VDU with an average luminance of $4.5 \mathrm{~cd} / \mathrm{m}^{2}$. The test stimulus was a $.5-\mathrm{cpd}$ sinusoidal grating in each experiment, and occupied a central $6^{\circ}$ diameter field. The test field was surrounded by a $13^{\circ}$ square field, the characteristics of which varied according to the experimental condition. Thresholds of gratings presented within the test area were obtained by means of the modified staircase method described previously (Bowling, 1985).

There were two subjects who participated in each of the three experiments, the author, A.C.B., and a research assistant, D.E.B., who was minimally familiar with the hypotheses of the experiments. Both subjects repeated each experimental condition five times, and each data point was the mean of eight threshold reversals. Each set of experimental conditions was preceded by a practice condition.

\section{EXPERIMENT 1}

The first experiment investigated the role of the contrast of a square-wave grating surround and the amplitude of modulation of a sinusoidally flickering surround upon the detection threshold of the .5-cpd test stimulus. It was hypothesized that increasing contrast or amplitude of modulation would initially produce an increase in threshold, but that further effects of increasing contrast would be minimal-that is, that the effect of contrast would saturate.

Bowling (1985) has shown that a horizontal surrounding grating has a smaller effect on the detection threshold of a vertical central test stimulus than a vertical grating. An additional horizontal surround was employed in the first experiment to investigate further differences in the effects produced by horizontal and vertical gratings on the detection threshold of the test. There were thus three surround conditions, a .65-cpd vertical square-wave grating drifting from right to left at $6.8 \mathrm{~Hz}$, the same grating oriented horizontally and moving upwards, and a blank field flickering sinusoidally at $6.8 \mathrm{~Hz}$. For each surround condition, six levels of contrast (or amplitude of flicker modulation) were employed, ranging from 0 to .6. The experiment was thus a 3 (surround condition) $\times 6$ (contrast) $\times 2$ (subjects) design.

\section{Results and Discussion}

The mean data for the two subjects are shown in Figure 1, and the combined data were analyzed by means
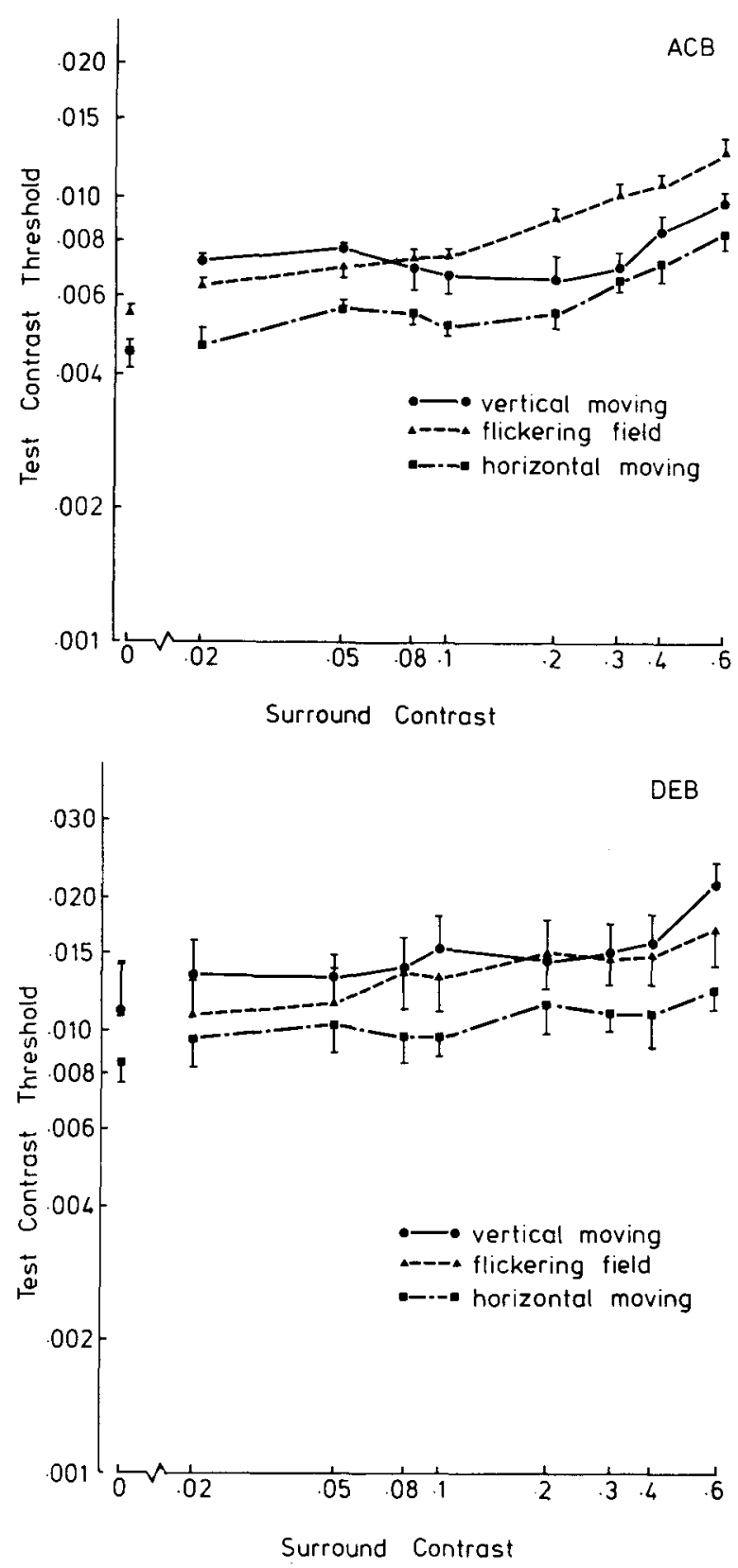

Figure 1. Graphs of the relationship between surround contrast (or depth of modulation) and the detection threshold of a central .5-cpd sinusoidal test grating counterphase flickering at 5 Hz. Surrounds were either vertical or horizontal $6.8-\mathrm{Hz}$ drifting .6-cpd square-wave gratings or a sinusoidally flickering blank field. Data for subjects A.C.B. and D.E.B. are shown separately.

of a subjects $x$ contrast $x$ surround analysis of variance. For each subject, there was an initial rise in the threshold of the test stimulus as the contrast of the surround increased. This was followed by a shallower, but continuing, rise with increasing surround contrast. There was a significant main effect of contrast $[F(8,216)=12.61$, $\mathrm{p}<.001]$, indicating that the detection threshold of the test grating is influenced by the surround contrast.

There was also a significant main effect of surround 
type $[\mathrm{F}(2,8)=28.93, \mathrm{p}<.001]$, indicating that the three types of surround influence detection threshold differently. This effect was largely due to the horizontal grating which had less of an effect upon threshold than either the vertical grating or flickering blank field across all contrast levels [these differences were both significant $(p<.001)$ ]. Thus, although the effect of the horizontal grating is significant $[F(8,72)=4.38, p<.001]$, it reduces test threshold to a much smaller extent than either the vertical or the flickering surround. This supports the data obtained previously (Bowling, 1985).

There was a significant main effect of subject (due to consistently lower thresholds for A.C.B. than for D.E.B.), and the surround $\times$ subject interaction was also significant $[\mathrm{F}(2,216)=5.21, \mathrm{p}<.01]$. This resulted from the two subjects' being influenced differently by the flickering and vertical moving surrounds. There was no significant contrast $\times$ subject interaction $[F(8,216)=0.71$, $\mathrm{p}>.05]$, indicating that there were no differences in the overall responses of the two subjects to the varying surround contrasts.

The data for both A.C.B. and D.E.B. show a continuing increase in test threshold with increasing surround contrast, although this increase is stronger for A.C.B. than for D.E.B. To assess the significance of this, a trend analysis was performed on the data for the two subjects separately. No significant deviations from linearity were observed with D.E.B.'s data $[F(7,108)=1.01, p>.05]$, but deviations were found with A.C.B.'s data, with comparisons of higher order than cubic being significant $[F(5,108)=3.73, p<.01]$. The shapes of the three functions obtained with the vertical moving, flickering, and horizontal moving surrounds were then analyzed separately to investigate the source of these deviations. The vertical surround condition was the only one showing a significant departure from linearity, there being significant trends higher than cubic $[F(5,108)=5.66, p<$ $.01]$. This is due to the rise and fall in test threshold with increasing contrast up to about 0.2 . This significant highorder nonlinear trend was not expected and may be due to a possible difficulty in discrimination of the test grating from the low-contrast vertical surround. This would then give rise to an artificially high test detection threshold at these low contrast levels.

The data do not show any evidence for a significant quadratic trend, which would be expected from the hypothesis that surround contrast has a saturating effect. The data consequently provide no evidence that increasing surround contrast fails to effect test threshold above a saturation point, and, consequently, the hypothesis that the effect is due to nonlinear subunits similar to those of the cat is not supported.

\section{EXPERIMENT 2}

In cats, the network of retinal subunits responds to spatial frequencies that are higher than those that give rise to the modulated response of $\mathrm{Y}$ cells (Hochstein \& Shapley, 1976). If a similar network is responsible for the threshold elevation of low-spatial-frequency gratings observed in the psychophysical experiments, this threshold elevation may also occur with higher surround spatial frequencies than those producing the transient response (i.e., above about $2 \mathrm{cpd}$ ). The second experiment consequently investigated the effect of the spatial frequency of the surround on the threshold elevation of the .5-cpd test grating.

There were eight surround spatial frequencies varying from $0 \mathrm{cpd}$ ( sinusoidally flickering blank field) to $8 \mathrm{cpd}$. All gratings were square-wave, with a contrast of .6 and a drift frequency of $6.8 \mathrm{~Hz}$. The blank field flickered at a rate of $6.8 \mathrm{~Hz}$, with an amplitude of modulation of .6 . A steady blank field surround condition was included as a control. A.C.B. and D.E.B. again served as subjects, and the data were analyzed with a 2 (subjects) $\times 9$ (spatial frequency) analysis of variance.

\section{Results and Discussion}

The data for the two subjects are shown in Figure 2. The analysis of variance showed that there were significant subject $[F(1,72)=76.18, p<.001]$ and spatial frequency $[F(8,72)=82.12, p<.001]$ main effects, as well as a subject $\times$ spatial frequency interaction $[F(8,72)=$ $3.00, p<.01]$. The subject main effect is due to the consistently higher thresholds produced by D.E.B. than by A.C.B., and the subject $\times$ frequency interaction to the high threshold produced by A.C.B. at $.5 \mathrm{cpd}$. The spatialfrequency main effect indicates that surround spatial frequency influences test threshold. Individual F comparisons between the baseline control mean and the other eight means were performed for each subject. For both A.C.B. and D.E.B., the mean test threshold for surround spatial frequencies of $0, .3, .5$, and $1 \mathrm{cpd}$ differed significantly (p $<.001$ ) from that of the control and the 3-, 4-, and 8 -cpd means were not significantly different $(p>.05)$. The 2-cpd mean differed significantly $(p<.01)$ from that of the control for A.C.B., but not for D.E.B. The data thus show that, under the conditions of this experiment, surround spatial frequencies greater than 2 cpd do not influence the detection thresholds of a central test grating, and, consequently, the subunit hypothesis is again not supported.

These data are basically the same as those obtained in an experiment by Green (1983), who investigated the effects of 7.2-Hz drifting sine-wave-grating surrounds upon a central flickering blank field. The elevation of the detection threshold of the temporal modulation of the central field declined with increasing surround spatial frequency, and was negligible at $3 \mathrm{cpd}$ and above. Consequently, it appears that the effects of moving surrounds are confined to spatial frequencies lower than 2 or $3 \mathrm{cpd}$.

In Experiment 2 the drift frequency of the moving surround was held constant. This means that, as spatial frequency increased, the velocity of the moving grating 


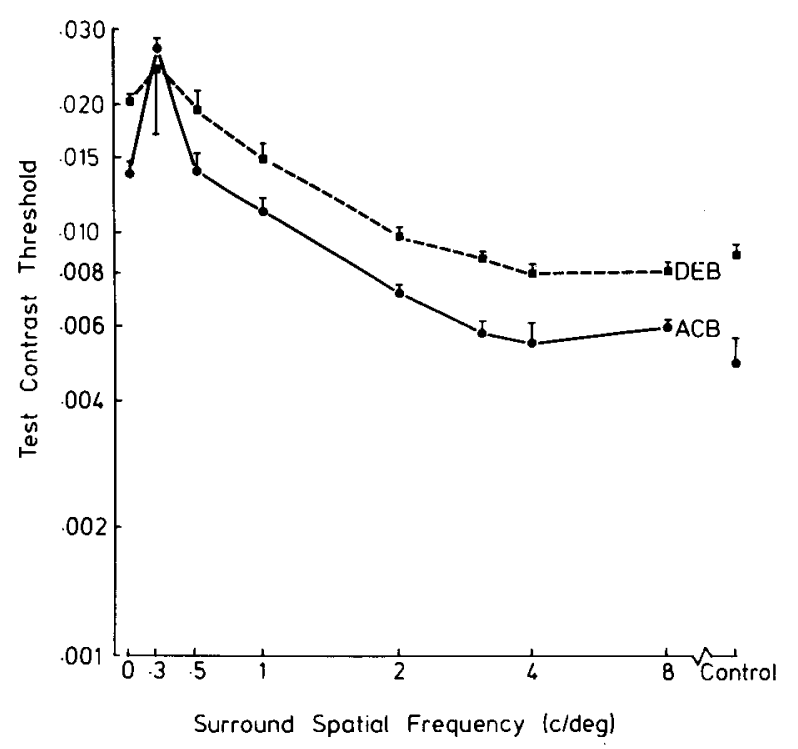

Figure 2. The relationship between surround spatial frequency and the detection threshold of the .5-cpd test stimulus for A.C.B. and D.E.B. as compared with a blank surround control condition.

decreased. Bowling (1985) has shown that the rate of motion of the surrounding grating influences sensitivity to the central test stimulus. However, it has not been established whether it is the temporal frequency or the velocity of the surround that is the crucial factor determining threshold reduction. If velocity is the important parameter, the spatial frequency effect observed in this experiment may be due to the decline in velocity with increasing spatial frequency. The third experiment investigated this possibility as part of an investigation of the effects of temporal frequency.

\section{EXPERIMENT 3}

Although the temporal frequency of the surround has been shown to influence the detection threshold of the central test stimulus, this effect has not been systematically investigated. It is also possible that the crucial surround temporal variable influencing test detection threshold is velocity rather than temporal frequency. If this is the case, then gratings moving with the same velocity but with differing spatial and temporal frequencies should have similar effects on the threshold of the test grating. Both spatial and temporal frequencies were varied in the third experiment to assess which of the factors of temporal frequency, spatial frequency, and velocity were the crucial ones affecting test threshold.

The temporal frequency of the surround was varied from 0 to $16 \mathrm{~Hz}$, with three surround spatial frequencies, $.5,1$, and $2 \mathrm{cpd}$. The same two subjects were employed, and the data were subjected to a 2 (subjects) $\times 7$ (temporal frequency) $\times 3$ (spatial frequency) analysis of variance.

\section{Results and Discussion}

The data for the two subjects are shown in Figure 3. It can be seen that threshold rises with increasing surround drift frequency to a maximum at $8 \mathrm{~Hz}$, but then declines at 12 and $16 \mathrm{~Hz}$. This occurs with both subjects and all three spatial frequencies. The greatest effects were observed with a surround spatial frequency of $.5 \mathrm{cpd}$; this was much reduced with $1 \mathrm{cpd}$. Although the figure indicates that there is some effect of the temporal frequency of the 2-cpd surround, this effect was not significant $[F(6,56)=1.19, p>.05]$. This observation confirms the finding of Experiment 2 that surrounds of $2 \mathrm{cpd}$ and
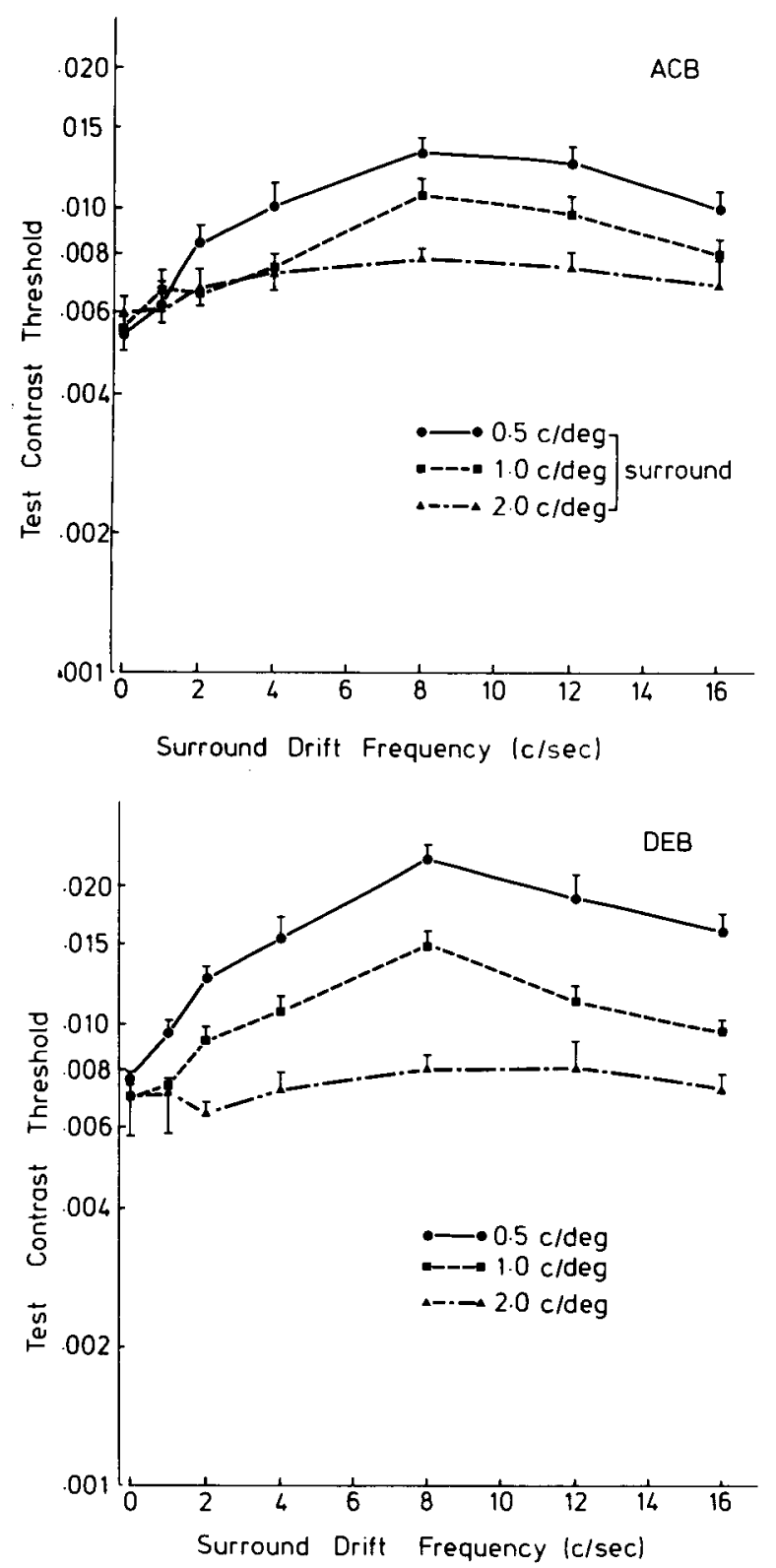

Figure 3. The relationship between surround drift frequency and test detection threshold for three surround spatial frequencies for A.C.B. and D.E.B. 
above have little influence on the detection threshold of the test grating, and shows that at these surround spatial frequencies, temporal modulation has a megligible effect.

The overall analysis of variance showed a significant subject main effect $[F(1,168)=65.84, p<.001]$ indicative of the higher thresholds of D.E.B. There were highly significant spatial frequency $[\mathrm{F}(2,168)=76.04, \mathrm{p}<$ $.001]$ and temporal frequency $[F(6,168)=31.64, p<$ $.001]$ main effects, indicating that both spatial and temporal frequencies have a role in affecting threshold. There was also a significant interaction between these two variables $[F(12,168)=4.10, p<.001]$ due to the fact that higher temporal frequencies are necessary to discriminate between the effects of the different spatial frequencies.

Comparisons of surrounds moving at the same velocity, but with differing spatial frequencies show that their threshold reduction effects are not constant, as would be expected if velocity alone controlled the effect, but decline with increasing spatial frequency. In addition, the observation that the effect of the surround temporal frequency peaks at $8 \mathrm{~Hz}$, regardless of spatial frequency, indicates that the temporal frequency of the surround, rather than velocity, is the important variable determining test threshold.

The results of this experiment consequently confirm that both the spatial and temporal frequencies of the surround play a role in affecting the detection threshold of the test grating.

\section{GENERAL DISCUSSION}

None of the experiments reported in this paper provide clear evidence for the hypothesis that a network of nonlinear subunits is responsible for the sensitivity reduction to a central test grating in the presence of a temporally modulated surround. There is no evidence for a saturating effect of surround contrast, or for an effect with higher spatial frequency surrounds. Both of these would have been expected if the mechanism responsible for the surround effects had properties similar to those of the subunits that apparently exist in the cat.

The three experiments have shown that both the rate of temporal modulation and the nature of the pattern of the surround influence the degree to which sensitivity to the central test pattern is reduced. Sensitivity is reduced to a much lesser extent when the surround is horizontal, and there is no effect of surround spatial frequencies greater than about $2 \mathrm{cpd}$. The effect increases with increasing temporal frequency to a maximum at about $8 \mathrm{~Hz}$, declining with temporal frequencies greater than this. These features indicate that the surround effect has similar characteristics to those of transient mechanisms. The visual system responds with transient time-courses to spatial frequencies lower than about 2 cpd (e.g. Green, 1981; Tolhurst, 1975), and also responds best to low-spatial-frequency gratings when these are temporally modulated at about $8-10 \mathrm{~Hz}$ (Kulikowski \& Tolhurst, 1973). Thus, optimal stimulation of transient mechanisms in the periphery by $8-\mathrm{Hz}$ modulation of low spatial frequencies has the maximal effect on the sensitivity of the mechanisms responsible for the detection of the test stimulus. It consequently appears that the threshold elevation of the low-frequency test stimuli is due to an inhibitory interaction between the peripheral and central transient mechanisms.

This interaction may be related to the masking effects observed when one flickering grating is detected in the presence of another (e.g., Kulikowski \& Gorea, 1978; Pantle, 1983). The detection threshold of a flickering test grating is elevated more by a flickering background grating than by a steady background. The logarithm of the threshold elevation is a linear function of the log background contrast with a slope of approximately 1 (Kulikowski \& Gorea, 1978). The relationship between $\log$ threshold elevation and log surround contrast for data from Experiment 1 was also approximately linear, but with a much shallower slope (approximately 0.2 ). It may thus be that activity in transient mechanisms in the periphery similarly masks that of central transient mechanisms but to a much weaker extent than when mask and test are spatially superimposed.

\section{REFERENCES}

Barlow, H. B., Derrington, A. M., Harris, L. R., \& Lennie, P. (1977). The effects of remote retinal stimulation on the responses of cat retinal ganglion cells. Journal of Physiology (London), 269, 177-194.

Bowling, A. C. (1985). The effects of peripheral movement and flicker on the detection thresholds of sinusoidal gratings. Perception \& Psychophysics, 37, 181-188.

Derrington, A. M., Lennie, P., \& Wright, M. J. (1979). The mechanisms of peripherally evoked responses in retinal ganglion cells. Journal of Physiology (London), 289, 299-310.

GrEEN, M. (1981). Spatial frequency effects in masking by light. Vision Research, 21, 861-866.

GrEEN, M. (1983). Visual masking by flickering surrounds. Vision Research, 23, 735-744.

Hochstein, S., \& Shapley, R. M. (1976). Linear and nonlinear spatial subunits in Y cat retinal ganglion cells. Joumal of Physiology (London), 262, 265-284.

KRUGER, J. (1980). The shift-effect enhances X-and suppresses Y-type response characteristics of cat retinal ganglion cells. Brain Research, 201, 71-84.

KRUGer, J. (1981). The difference between X-and Y-type responses in ganglion cells of the cat's retina. Vision Research, 21, 1685-1687.

Kulikowski, J. J., \& GOREA, A. (1978). Complete adaptation to patterned stimuli: A necessary and sufficient condition for Weber's law for contrast. Vision Research, 18, 1223-1227.

KulikowsKI, J. J., \& Tolhurst, D. J. (1973). Psychophysical evidence for sustained and transient detectors in human vision. Journal of Physiology, 232, 149-162.

Pantle, A. J. (1983). Temporal determinants of spatial sine-wave masking. Vision Research, 23, 749-757.

TolHURST, D. J. (1975). Reaction times in the detection of gratings by human observers: A probabalistic mechanism. Vision Research, 15, 1143-1149.

\section{NOTE}

1. In this paper, as in the previous one, the terms "transient" and "sustained" will be used to refer to the shape of the response timecourse. "Transient" refers to a time-course with pronounced activity at stimulus on- and offset, as opposed to "sustained," where activity occurs throughout stimulus presentation. It does not assume that separate "pattern" and "flicker" mechanisms exist across the spatial frequency spectrum.

(Manuscript received October 1, 1984; revision accepted for publication October 10,1985 .) 